\title{
Creatividad, Emoción y Espacio: El mundo a través de la experiencia sensible Proyecto de Investigación
}

$\mathrm{N}^{\circ} 3.7$

Equipo de Investigación ${ }^{(1)}$

Sandra Navarrete

por Universidad de Mendoza (ARG) y

Facultad de Diseño y Comunicación, Universidad de Palermo (ARG)

Resumen: El Proyecto de Investigación 3.7 fue concebido desde tres posibles abordajes del diseño: Creatividad, Emoción y Espacio. Creatividad, palabra frecuentemente mencionada en los ámbitos académicos y profesionales del diseño. Emoción, concepto vinculado a disciplinas de las ciencias sociales, incluso de las ciencias de la salud, pero en la investigación científica del diseño aparece como algo menos riguroso, es un riesgo que pocas veces se acepta asumir. Espacio, el objeto de todo estudio relacionado con el interiorismo, la arquitectura y otras disciplinas proyectuales. Tres ideas diferentes, es posible encontrar las zonas de contacto entre ellas?... y en ese caso por qué nos hemos propuesto este desafío? Se propone consolidar una investigación (a nivel regional, latinoamericano, internacional) que incorpore la perspectiva fenomenológica, en el ámbito académico y en la práctica del diseño. Es un apasionante camino desde lo sensorial, emocional, humanizado hacia las mejores respuestas creativas que pueden aportarse al mundo actual.

Palabras clave: Investigación fenomenológica - Diseño emocional - Creatividad - Didáctica proyectual.

[Resúmenes en inglés y portugués en la página 116]

(1) Los CVs del Equipo de Investigación pueden consultarse en el Capítulo Directores de Lineas y Coordinadores de Proyectos de esta misma Edición.

\section{Acerca del Proyecto 3.7}

Creatividad, Emoción y Espacio

El mundo a través de la experiencia sensible

El Proyecto 3.7 Creatividad, Emoción y Espacio: El mundo a través de la experiencia sensible, aborda la comprensión del espacio desde la concepción fenomenológica en la 
que se incorporan los modos de aprehender el mundo a través de la experiencia sensible. En la práctica proyectual se involucran experiencias estéticas, sensibles, para lograr una aproximación integradora y humanizada a la idea generadora de la obra a diseñar. Se desarrollan tres posibles abordajes: Creatividad, mencionada en los ámbitos académicos y profesionales del diseño; Emoción, vinculada a disciplinas de las ciencias sociales y ciencias de la salud, pero en la investigación científica del diseño aparece como algo menos riguroso, es un riesgo que pocas veces se acepta asumir; y Espacio, objeto de todo estudio relacionado con el interiorismo, la arquitectura y disciplinas proyectuales ¿Es posible encontrar zonas de contacto entre ellas? El objetivo es consolidar y desarrollar una temática de gran interés que en la actualidad se encuentra dispersa, para alcanzar mayor profundidad desde una mirada multidisciplinar.

Se acordó entre la Universidad de Palermo (Argentina) y la Universidad de Mendoza (Argentina) avanzar en una investigación en la que participan académicos e Invitados de estas y otras Instituciones, con la coordinación de Sandra Navarrete, para consolidar un Proyecto de investigación a nivel regional, latinoamericano, e internacional que incorpora la perspectiva fenomenológica al ámbito académico y en la práctica del Diseño.

Las reflexiones y los resultados obtenidos en el Proyecto 3.7 Creatividad, Emoción y Espacio son continuación de los Proyectos 3.2 Morfología espacial y objetual I, 3.4 Morfología espacial y objetual II y 3.6 Morfología espacial y objetual III, y, asimismo guarda relación y comparte alguno de sus investigadores con los Proyectos 3.1 Materialidad Difusa, 3.3: Identidad Difusa y 3.5 Profesionalización Difusa en el marco de la misma Línea de Investigación y bajo la misma Directora Ana Cravino.

Sus principales objetivos son:

- Reflexionar desde distintas experiencias y enfoques la dimensión emocional y sensorial, en la producción artística, proyectual y cultural contemporánea.

- Estudiar la fenomenología proyectual desde la propia concepción esencial de lo subjetivo, con énfasis en tres ejes: la cuestión sensorial dentro de las experiencias pedagógicas de diseño, en las experiencias proyectuales de integración artística, técnica y/o expresiva y en las reflexiones teóricas y metodológicas sobre Creatividad, Emoción y Espacio.

- Indagar sobre los procesos proyectuales en las fronteras donde otras disciplinas como la filosofía y la psicología, que vienen aportando a la comprensión de esta forma de comprender el diseño.

\section{Acerca de la Línea 3}

Forma y Materialidad: Incidencia de la morfología y la tecnología en el diseño de espacios y productos, dirigida por Ana Cravino se desarrolla de manera ininterrumpida desde 2015 en la Facultad de Diseño y Comunicación (UP, Argentina), e incluye hasta el momento siete proyectos finalizados, el 3.1 Materialidad Difusa y 3.3 Identidad Difusa coordinados por Daniel Wolf (UP) y Luisa Collina (Instituto Politécnico de Milán, 
Italia); el 3.5 Profesionalización Difusa coordinado por Fabiola Knop y Alejo García de la Cárcova (UP) junto a la Universidad Nacional de Mar del Plata (Argentina) y la Universidad Rafael Landivar (Guatemala); el 3.2 Morfología espacial y objetual I coordinado por Ana Cravino y Roberto Céspedes (UP); el 3.4 Morfología espacial y objetual II coordinado por Ana Cravino y Roberto Céspedes (UP), Taña Escobar Guanoluisa (Universidad Técnica de Ambato, Ecuador) y César Giovanny Delgado Banegas (Universidad del Azuay, Ecuador); el 3.6 Morfología espacial y objetual III coordinado por Ana Cravino (UP) y Genoveva Malo (Universidad del Azuay, Ecuador); y el 3.7 Creatividad, Emoción y Espacio coordinado por Sandra Navarrete (Universidad de Mendoza, Argentina).

\section{Mapa de Áreas y Proyectos}

El Proyecto 3.7 se vincula con todas las carreras de grado y posgrado correspondientes a la Facultad de Diseño y Comunicación, y específicamente Diseño de Interiores, Diseño de Moda y Diseño Industrial de la propia unidad académica y la carrera de Arquitectura de la Facultad del mismo nombre.

\section{Productos y Resultados}

\section{a)- Publicaciones}

Cuadernos del Centro de Estudios de Diseño y Comunicación No109. (2020/2021) Creatividad, Emoción y Espacio. Coordinación: Sandra Navarrete. Año XXIV, Buenos Aires, Argentina. ISSN: 1668-0227. Esta publicación documenta y comunica los resultados alcanzados en el Proyecto 3.7 Creatividad, Emoción y Espacio y a continuación se detallan los autores $y$ artículos contenidos en ella:

Navarrete, Sandra (2020/2021) Prólogo. Creatividad, Emoción y Espacio (Pp.1319)

Asarchuk, Andrés Gustavo; Fernández Rabadán, María Macarena (2020/2021) De la representación a los hallazgos (Pp.20-31)

McDonough, Peggy; Bermúdez, Julio (2020/2021) Abstracción, Transformación, e Inspiración. El arte como fuente de conocimiento y creatividad arquitectónica en el taller de diseño (Pp.33-47)

Donoso, Sergio; Vielma, Mitzi (2020/2021) Imágenes y palabras afectivas para estimular la imaginación: un caso en la didáctica proyectual (Pp.49-61)

Ojeda, Beatriz Eugenia; Escoriaza Nasazzi, María Jimena (2020/2021) Conversaciones acerca del espacio. Argumentaciones teóricas desde el hacer para el hacer (Pp.63-72)

Giani, Esther (2020/2021) La belleza como valor emocional en el espacio cultural (Pp.72-85)

Medina, Gabriel Leonardo (2020/2021) Secuencias didácticas en la dimensión del espacio: prototipando mapas gráfico-espaciales (Pp.85-104) 
Tonelli, Inés (2020/2021) Exploraciones en el campo de la investigación proyectual fenomenológica. La cuestión sensorial en las experiencias pedagógicas de diseño (Pp.105-116)

Bianchi, Pablo (2020/2021) La fenomenología de la percepción como estrategia de enseñanzaaprendizaje del proceso proyectual en arquitectura. La cuestión sensorial (Pp.117-127)

Serrani, Sebastián; Escoriaza, Jimena (2020/2021) Hacia una arquitectura análoga de la música. Principios, lógicas metodológicas y presentación de casos (Pp.129152)

Álvarez Falcón, Luis (2020/2021) Reproducibilidad y el diseño de las imágenes, de los espacios, de los tiempos y de los afectos (Pp.153-161)

Pereyra de Andrade, Ana Beatriz; Rebello Magalhaes, Ana Maria; Franco Mathias, Ariadne; Furio Melara, Lucas; Rebello Magalhaes de Oliveira, Paula (2020/2021) Desenhando Memórias em Espaços Solidários: um registro de emoções no abrigo de idosos Vila Vicentina (Pp. 163-177)

Montaño Lozano, Édgar (2020/2021) El juego consciente en el proceso del pensamiento creativo. Desde las emociones para no dejar de sentir tu ser (Pp. 179-187)

Navarrete, Sandra (2020/2021) Los espacios del silencio. Una reflexión de base fenomenológica de lo no evidente en la arquitectura (Pp.189-202)

Brkljacic, Marcela (2020/2021) Desde la crisis existencial a la sustancia de la arquitectura. Relatos sobre el vacío (Pp.203-211)

Serrani, Sebastián (2020/2021) El recorrido en la arquitectura y ciudad una aproximación desde la crítica y el relato (Pp.213-222)

\section{b)- Congresos / Coloquios / Plenarios}

V Coloquio de Investigación y Desarrollo en Diseño Latino. Universidad de Palermo, 27 de julio de 2020. XI Congreso Latinoamericano de Enseñanza del Diseño. Semana Internacional del Diseño en Palermo. Se presentaron los resultados y avances de la Línea de Investigación No3: Forma y Materialidad en tres comisiones Realidad Difusa, Taxonomías Espaciales y Objetuales, y Creatividad, Emoción y Espacio.

- En la Comisión Creatividad, Emoción y Espacio se presentaron las reflexiones y conclusiones del proyecto 3.7 Creatividad, Emoción y Espacio (Finalizado) expusieron: Andrés Asarchuk, Macarena Fernández Rabadán, Peggy McDonough, Julio Bermudez, Sergio Donoso, Mitzi Vielma, Beatriz Eugenia Ojeda María, Jimena Escoriaza Nasazzi, Esther Gianni, Gabriel Leonardo Medina, Inés Tonelli, Pablo Bianchi, Sebastián Serrani, Jimena Escoriaza, Luis Álvarez Falcón, Ana Beatriz Pereira de Andrade, Ana Maria Rebello Magalhães, Ariadne Franco Mathias, Lucas Furio Melara, Paula Rebello Magalhães de Oliveira, Édgar Montaño Lozano, Marcela Brkjljacic, Sebastián Serrani y Sandra Navarrete.

A continuación se detallan las ponencias presentadas en esta Comisión coordinada por los Directores del Proyecto Sandra Navarrete y Sebastián Serrani: 
Andrés Asarchuk y Macarena Fernández Rabadán (Argentina)

De la representación a los hallazgos

Peggy McDonough y Julio Bermudez (USA)

Abstracción, Transformación, e Inspiración. El arte como fuente de conocimiento y creatividad arquitectónica en el taller de diseño

Sergio Donoso y Mitzi Vielma (Chile)

Imágenes y palabras afectivas para estimular la imaginación: un caso en la didáctica proyectual

Beatriz Eugenia Ojeda María y Jimena Escoriaza Nasazzi (Argentina)

Conversaciones acerca del espacio

Esther Gianni (Italia)

La belleza como valor emocional en el espacio cultural

Gabriel Leonardo Medina (Argentina)

Secuencias didácticas en la dimensión del espacio

Inés Tonelli (Argentina)

Exploraciones en el campo de los modos de pensamiento del diseñador y lógicas proyectuales capaces de introducir ideas creativas en el proceso de diseño

Pablo Bianchi (Argentina)

La fenomenología de la percepción como estrategia de enseñanza-aprendizaje del proceso proyectual en arquitectura. La cuestión sensorial en las experiencias pedagógicas de diseño

Sebastián Serrani y Jimena Escoriaza (Argentina)

Hacia una arquitectura análoga de la música. Principios, lógicas metodológicas y presentación de casos

Luis Álvarez Falcón (España)

Fenomenología del diseño: la reproducibilidad y el diseño de las imágenes, de los espacios, de los tiempos $y$ de los afectos

Ana Beatriz Pereira de Andrade, Ana Maria Rebello Magalhães, Ariadne Franco Mathias, Lucas Furio Melara y Paula Rebello Magalhães de Oliveira (Brasil)

Desenhando Memórias em Espaços Solidários: um registro de emoções no abrigo de idosos Vila Vicentina

Édgar Montaño Lozano (Colombia)

El juego consciente en el proceso del pensamiento creativo. Desde las emociones para no dejar de sentir tu ser por medio del crear/ conectar/ comprender/ construir Marcela Brkjljacic (Argentina)

Desde la crisis existencial a la sustancia de la arquitectura. Relatos sobre el vacío Sebastián Serrani (Argentina)

El recorrido en la arquitectura y ciudad una aproximación desde la crítica y el relato Sandra Navarrete (Argentina)

Los espacios del silencio. Una reflexión sobre lo no evidente en la arquitectura 


\title{
c)- Formación de Posgrado e Impacto curricular
}

La Directora Ana Cravino es Docente de Posgrado en Diseño de la Facultad de Diseño y Comunicación en Maestría en Gestión del Diseño y Doctorado en Diseño y al igual que Sandra Navarrete en su Institución (Universidad de Mendoza, Argentina), ambas incorporan los contenidos de su investigación a sus asignaturas en posgrado.

\begin{abstract}
Research Project 3.7 was conceived from three possible design approaches: creativity, emotion and space. Creativity, a word frequently mentioned in the academic and professional fields of design. Emotion, a concept linked to disciplines of the social sciences, including the health sciences, but in scientific design research it appears as something less rigorous, it is a risk that is rarely accepted to assume. Space, the object of any study related to interior design, architecture and other design disciplines. Three different ideas, is it possible to find the contact areas between them?... and in that case, why have we set ourselves this challenge? It is proposed to consolidate an investigation (at the regional, Latin American, international level) that incorporates the phenomenological perspective, in the academic field and in the practice of design. It is an exciting path from the sensory, emotional, humanized to the best creative responses that can be brought to today's world.
\end{abstract}

Keywords: Phenomenological research - Emotional design - Creativity - Project didactics.

Resumo: O Projeto de Pesquisa 3.7 foi concebido a partir de três abordagens de design possíveis: criatividade, emoção e espaço. Criatividade, palavra frequentemente mencionada no meio acadêmico e profissional do design. Emoção, conceito vinculado às disciplinas das ciências sociais, incluindo as ciências da saúde, mas na pesquisa em design científico aparece como algo menos rigoroso, é um risco que raramente se aceita assumir. Espaço, objeto de qualquer estudo relacionado ao design de interiores, arquitetura e outras disciplinas de design. Três ideias diferentes, é possível encontrar as áreas de contacto entre elas? ... e nesse caso, porque nos propusemos este desafio? Propõe-se consolidar uma investigação (em nível regional, latino-americano, internacional) que incorpore a perspectiva fenomenológica, no campo acadêmico e na prática do design. É um caminho estimulante desde o sensorial, emocional, humanizado até as melhores respostas criativas que podem ser trazidas ao mundo de hoje.

Palavras chave: Pesquisa fenomenológica - Desenho emocional - Criatividade - Didática de projetos.

[Las traducciones de los resúmenes fueron realizadas a través de traductor automático] 